BMC

Microbiology

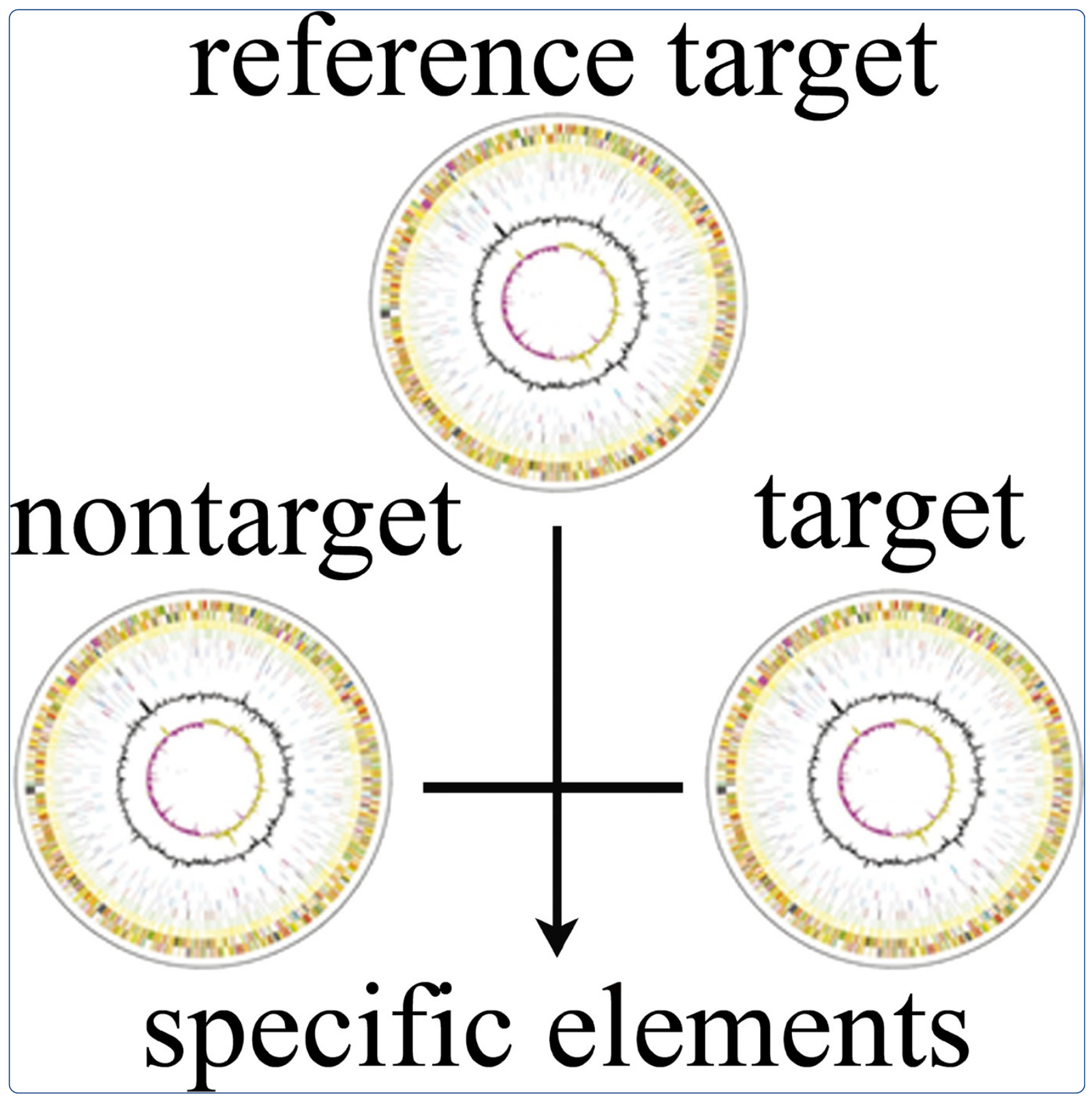

atpE gene as a new useful specific molecular target to quantify Mycobacterium in environmental samples

Radomski et al.

() Biomed Central 


\title{
atpE gene as a new useful specific molecular target to quantify Mycobacterium in environmental samples
}

\author{
Nicolas Radomski ${ }^{1,2^{*}+}$, Adélaïde Roguet ${ }^{1}$, Françoise S Lucas ${ }^{1 \dagger}$, Frédéric J Veyrier ${ }^{3}$, Emmanuelle Cambau ${ }^{4 \dagger}$,
} Héberte Accrombessi ${ }^{5}$, Régis Moilleron ${ }^{1 \dagger}$, Marcel A Behr $^{2}$ and Laurent Moulin ${ }^{5+}$

\begin{abstract}
Background: The environment is the likely source of many pathogenic mycobacterial species but detection of mycobacteria by bacteriological tools is generally difficult and time-consuming. Consequently, several molecular targets based on the sequences of housekeeping genes, non-functional RNA and structural ribosomal RNAs have been proposed for the detection and identification of mycobacteria in clinical or environmental samples. While certain of these targets were proposed as specific for this genus, most are prone to false positive results in complex environmental samples that include related, but distinct, bacterial genera. Nowadays the increased number of sequenced genomes and the availability of software for genomic comparison provide tools to develop novel, mycobacteria-specific targets, and the associated molecular probes and primers. Consequently, we conducted an in silico search for proteins exclusive to Mycobacterium spp. genomes in order to design sensitive and specific molecular targets.
\end{abstract}

Results: Among the 3989 predicted proteins from M. tuberculosis H37Rv, only 11 proteins showed 80\% to 100\% of similarity with Mycobacterium spp. genomes, and less than 50\% of similarity with genomes of closely related Corynebacterium, Nocardia and Rhodococcus genera. Based on DNA sequence alignments, we designed primer pairs and a probe that specifically detect the atpE gene of mycobacteria, as verified by quantitative real-time PCR on a collection of mycobacteria and non-mycobacterial species. The real-time PCR method we developed was successfully used to detect mycobacteria in tap water and lake samples.

Conclusions: The results indicate that this real-time PCR method targeting the atpE gene can serve for highly specific detection and precise quantification of Mycobacterium spp. in environmental samples.

Keywords: Mycobacteria, atpE gene, Environmental samples

\section{Background}

Mycobacterium genus is constituted of several pathogenic species, including the $M$. tuberculosis complex (MTC) responsible for tuberculosis (i.e. M. tuberculosis, $M$. africanum, $M$. bovis, $M$. canettii, $M$. caprae, $M$. microti and $M$. pinnipedii), $M$. leprae responsible for

\footnotetext{
* Correspondence: nicolas.radomski.phd@gmail.com

${ }^{\dagger}$ Equal contributors

'Laboratoire Eau Environnement Systèmes Urbains (Leesu) UMR MA 102-AgroParisTech, Université Paris-Est, 6-8 avenue Blaise Pascal Cité Descartes, FR 77455, Champs sur Marne, France

${ }^{2}$ Research Institute of the McGill University Health Centre, 1650 Cedar

Avenue, Montreal H3G 1A4, QC, Canada

Full list of author information is available at the end of the article
}

leprosy, and non-tuberculous mycobacteria (NTM), which are environmental potentially pathogenic species causing mycobacteriosis [1]. Detection of mycobacteria by bacteriological tools is generally time-consuming and difficult because most pathogenic mycobacteria are slow growing, such that other microorganisms overgrow NTM colonies [2]. Identification of mycobacteria based on metabolic criteria is also problematic as current methods do not allow for proper identification of mycobacterial species and subspecies. Consequently, molecular tools have been developed using $r r s, g y r A, g y r B, h s p 65$, recA, rpoB, sodA genes and 16S-23S internal transcribed spacer (ITS) genes, to detect and/or identify mycobacteria species by sequence 
analysis $[3,4]$. In order to detect Mycobacterium genus in clinical and environmental samples, several studies have proposed targeting different loci of the $16 \mathrm{~S}$ rRNA gene [5-17], or other housekeeping genes such as $\operatorname{gyr} B$ [18], $r p o B$ [19], and $h s p 65$ [20]. Nevertheless, in a recent study comparing several primers commonly used for mycobacterial detection or identification, we demonstrated that most of these primers present either a high specificity (i.e. the proportion of true negatives that are correctly identified by the test) but a low sensitivity (i.e. the proportion of true positives that are correctly identified by the test), or conversely a high sensitivity but a low specificity [17]. Indeed, some of these methods fail to detect several mycobacterial species by PCR, while other primers lead to detection of closely related genera [17] which also belong to the Corynebacterium, Nocardia, Rhodococcus, Mycobacterium (CNM) group [21] and which are commonly present in water and soil samples. Consequently, new strategies must be used in order to design Mycobacterium genus targets with high levels of specificity and sensitivity that will be useful for studying mycobacteria in their habitat.

As new mycobacterial sequences are added into genetic databases, our knowledge of mycobacterial genomes is increasing and this may help to design new primers and probes that will be both specific and sensitive. Since the whole sequencing of the first mycobacterial genome in 1998 [22] by Sanger sequencing method (M. tuberculosis H37Rv), the number of mycobacterial sequences has considerably increased due to advances in sequencing capacity and the appearance of high throughput sequencing techniques [23]. Today, GenBank database provides access to whole genomes of seven other strains of the MTC $(M$. tuberculosis and $M$. bovis species), two strains of $M$. leprae, and eleven species and subspecies of pathogenic (P) and non-pathogenic (NP) NTM: M. abscessus (P), M. avium (P), M. avium subsp. paratuberculosis (P), M. gilvum (NP), M. marinum (P), M. smegmatis (NP), Mycobacterium sp. JLS (NP), Mycobacterium sp. KMS (NP), Mycobacterium sp. MCS (NP), M. ulcerans (P), M. vanbaalenii (NP), [24-26]. Moreover, three whole genomes of other NTM species were sequenced and are currently assembled $(M$. intracellulare, M. kansasii, M. parascrofulaceum). This increasing number of completely sequenced mycobacterial genomes led to the development of the MycoHit software, which permits gene- and protein-level comparisons across mycobacteria species, [27]. This software was originally developed to detect horizontal gene transfers and mutations among whole mycobacterial genomes [27]. However, MycoHit should also be useful for developing new primers and probes for mycobacteria detection and quantification in environmental and clinical samples.

In this paper, we used this tool for screening sensitive and specific targets of Mycobacterium spp.. We compared in silico proteins of whole mycobacterial genomes with those of non-mycobacterial genomes using the MycoHit software, in order to find conserved sequences among mycobacteria that will not be shared with non-mycobacterial species. Based on the screening results a primer pair and a probe targeting the $a t p E$ gene were designed and tested by realtime PCR. This novel target proved to be totally specific and sensitive. It also offers the advantage of targeting a gene present as a single copy in the genome. Thus this new realtime PCR method appears promising for water quality survey, and should be useful for studying the ecology of mycobacteria in aquatic, terrestrial and urban environments.

\section{Results}

\section{Specificity of genes commonly used for mycobacterial detection/identification}

Excluding rrs gene and ITS (non-functional RNA elements and structural ribosomal RNAs), and according to our strategy of genome comparison (Figure 1) most of the genes commonly used for mycobacterial species identification ( $g y r A, \operatorname{gyr} B, h s p 65, \operatorname{rec} A, \operatorname{rpoB}, \operatorname{sod} A, \operatorname{groEL1}$, groEL2) code for proteins which present similar conformations in non-mycobacterial studied genomes (Additional file 1). Indeed, protein similarity levels of these genes, in comparison with $M$. tuberculosis $\mathrm{H} 37 \mathrm{Rv}$ genome, were higher than $80 \%$ for the other 15 mycobacterial genomes studied $(96 \pm 2 \%$ for $\operatorname{gyr} A, 94 \pm 5 \%$ for $\operatorname{gyr} B, 79 \pm 5 \%$ for groEL1, $93 \pm 4 \%$ for groEL2 which is an alternative gene name for $h s p 65,99 \pm$ $1 \%$ for $r e c A, 96 \pm 2 \%$ for $r p o B, 81 \pm 33 \%$ for $\operatorname{sod} A$ ), and also for the 12 non-mycobacterial genomes studied ( $86 \pm 5 \%$ for $\operatorname{gyr} A, 85 \pm 5 \%$ for $g y r B, 89 \pm 3 \%$ for $\operatorname{gro} E L 1,96 \pm 2 \%$ for groEL2, $94 \pm 3 \%$ for $r e c A, 88 \pm 4 \%$ for $r p o B, 69 \pm 22 \%$ for $\operatorname{sod} A)$.

\section{Selection of exclusively conserved proteins in Mycobacterium spp. genomes}

Among the 3989 predicted proteins of M. tuberculosis H37Rv genome (Figure 2A and Additional file 1), about $54.6 \%$ (i.e. 2177 proteins) presented protein similarities above $50 \%$ with the other studied mycobacterial genomes $(\mathrm{n}=15)$, and only $6.8 \%$ of these hypothetical conserved mycobacterial proteins (150 proteins: 150 number in the top of a bar in Figure 2B) displayed similarities less than $50 \%$ with the studied non-mycobacterial genomes $(n=12)$. Consequently, almost half of the M. tuberculosis $\mathrm{H} 37 \mathrm{Rv}$ predicted proteins are potentially present in the 12 studied genomes of CNM group members. We chose to decrease the number of candidate proteins by restricting the panel of studied proteins to those exclusively conserved in the mycobacterial genomes, focusing on $M$. tuberculosis H37Rv proteins with similarity levels between $80 \%$ and $100 \%$ in comparison with other mycobacterial genomes $(\mathrm{n}=15)$, and less than $50 \%$ similarity levels in comparison with genomes $(n=12)$ of the other CNM group genera. As a result, among the 3989 predicted proteins of $M$. 

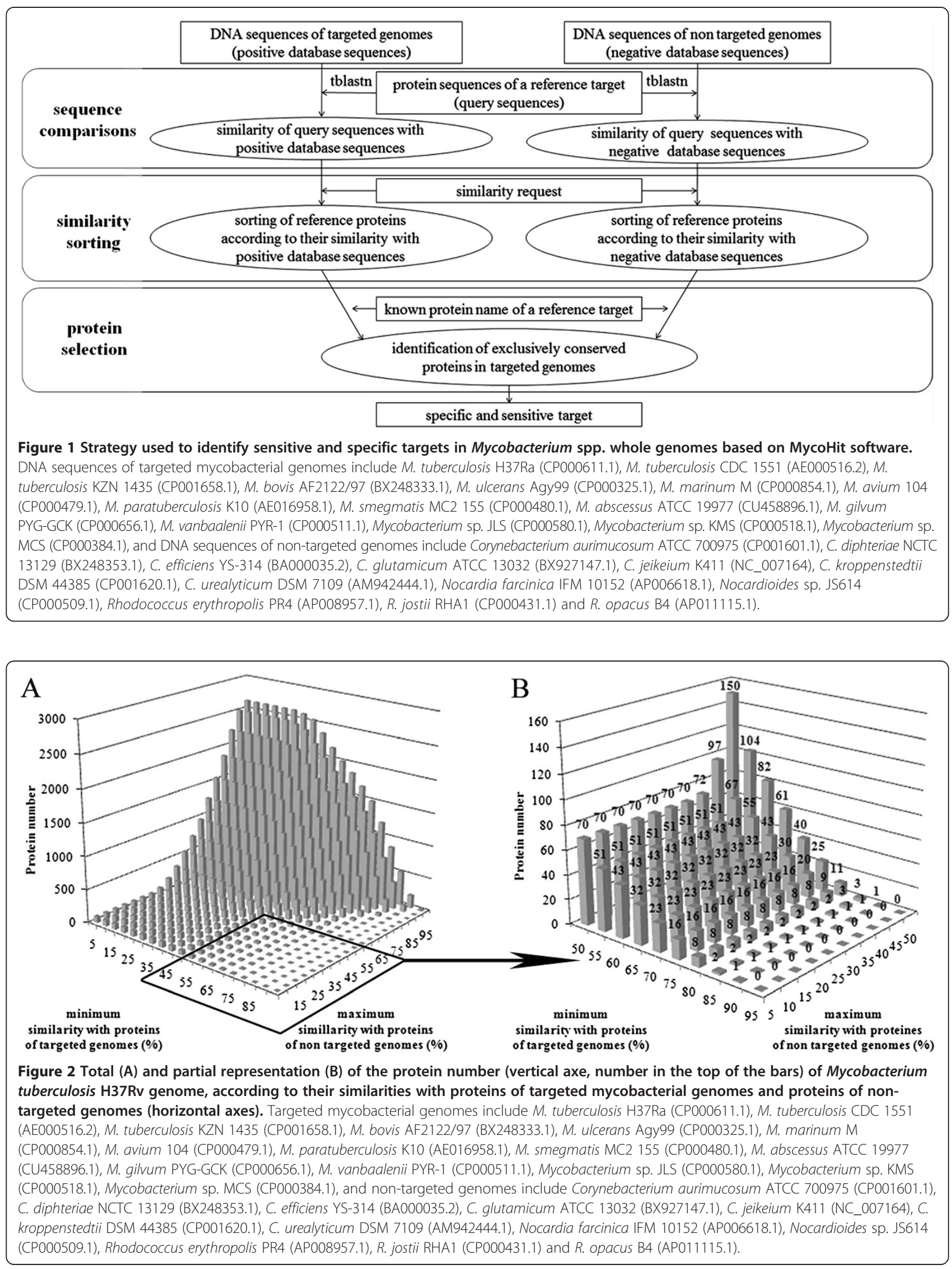
tuberculosis H37Rv genome (Figure 2A), we selected 11 proteins (11 number in the top of a bar in Figure 2B). Among the 3989 predicted proteins of $M$. tuberculosis H37Rv proteins (Additional file 1), the selected candidate proteins (Table 1), were the subunits C (locus Rv1305) and A (locus Rv1304) of the ATP synthase, the cyclopropane mycolic acid synthase (CMAS) coded by the cmaA1 gene in M. tuberculosis H37Rv (locus Rv3392c), hypothetical PE or PPE family proteins (loci Rv0285 and Rv3022c), proteins coded by esxG, esxH and esxR genes in M. tuberculosis H37Rv (loci Rv0287, Rv0288, Rv3019c, respectively), and proteins such as a lipoprotein coding by $\operatorname{lpp} M$ gene (locus Rv2172c), an oxidoreductase (locus Rv0197), and a small secreted protein (locus Rv0236A).

\section{Mycobacterial molecular target design}

Among the 11 selected mycobacterial proteins, protein alignments revealed that the ATP synthase subunit C (locus Rv1305), the oxidoreductase (locus Rv0197), and the small secreted protein (locus Rv0236A), are the less polymorphous among the 14 NTM species studied (Additional file 2) and even absent in other bacteria genus and thus seemed very promising for primers and probes design. The remaining 8 proteins that were selected, namely ATP synthase subunit A, CMAS coded by the $c m a A 1$ gene, lipoprotein coding by $\operatorname{lpp} M$ gene, as well as PE, PPE and proteins coded by es $x$ genes es $x G$, $e s x H$ and $e s x R$, were highly conserved in studies MTC species (tuberculosis and bovis) but very polymorphous

Table 1 Similarity (\%) of the most conserved mycobacterial proteins in Mycobacterium spp., Corynebacterium spp., Nocardia spp. and Rhodococcus spp. genomes, in comparison with M. tuberculosis H37Rv genome

\begin{tabular}{|c|c|c|c|c|c|c|c|c|c|c|c|}
\hline Protein locus (H37Rv genome) & Rv1305 & Rv0236A & Rv0197 & Rv2172c & Rv0287 & Rv0288 & Rv3019c & Rv0285 & Rv3022c & Rv1304 & Rv3392c \\
\hline protein length (aa) & 81 & 57 & 762 & 301 & 97 & 96 & 96 & 102 & 81 & 250 & 287 \\
\hline gene name & atpE & - & - & IppM & esxG & esxH & esxR & PE5 & PPE48 & $\operatorname{atpB}$ & $c m a A 1$ \\
\hline M. tuberculosis H37Ra & 100 & 100 & 99 & 100 & 100 & 100 & 100 & 100 & 100 & 100 & 100 \\
\hline M. tuberculosis CDC1551 & 100 & 100 & 99 & 100 & 100 & 100 & 100 & 100 & 100 & 100 & 99 \\
\hline M. tuberculosis KZN 1435 & 100 & 100 & 99 & 100 & 100 & 100 & 100 & 100 & 100 & 100 & 100 \\
\hline M. bovis AF2122/97 & 100 & 100 & 99 & 100 & 100 & 100 & 100 & 100 & 98 & 100 & 100 \\
\hline M. ulcerans Agy99 & 100 & 96 & 86 & 90 & 96 & 92 & 93 & 93 & 83 & 96 & 87 \\
\hline M. marinum M & 100 & 98 & 90 & 91 & 96 & 89 & 94 & 93 & 82 & 97 & 88 \\
\hline M. avium 104 & 96 & 96 & 91 & 91 & 91 & 89 & 91 & 92 & 83 & 93 & 82 \\
\hline M. paratuberculosis K10 & 96 & 96 & 91 & 91 & 91 & 89 & 91 & 92 & 85 & 92 & 82 \\
\hline M. smegmatis MC2 155 & 93 & 91 & 85 & 83 & 87 & 85 & 85 & 87 & 82 & 84 & 86 \\
\hline M. abscessus ATCC 19977 & 98 & 85 & 85 & 82 & 81 & 81 & 80 & 82 & 81 & 85 & 82 \\
\hline M. gilvum PYR-GCK & 100 & 91 & 85 & 86 & 88 & 88 & 85 & 85 & 80 & 83 & 81 \\
\hline M. vanbaalenii PYR-1 & 93 & 91 & 85 & 87 & 89 & 85 & 83 & 82 & 83 & 84 & 81 \\
\hline Mycobacterium sp. JLS & 100 & 91 & 85 & 86 & 87 & 86 & 86 & 82 & 82 & 89 & 92 \\
\hline Mycobacterium sp. KMS & 100 & 91 & 86 & 86 & 88 & 86 & 86 & 82 & 82 & 89 & 91 \\
\hline Mycobacterium sp. MCS & 100 & 91 & 86 & 86 & 88 & 86 & 86 & 82 & 82 & 89 & 91 \\
\hline C. aurimucosum ATCC 700975 & 0 & 0 & 0 & 0 & 0 & 0 & 0 & 0 & 0 & 0 & 46 \\
\hline C. diphteriae NCTC 13129 & 0 & 0 & 0 & 0 & 0 & 0 & 0 & 0 & 0 & 43 & 0 \\
\hline C. efficiens YS-314 & 0 & 0 & 42 & 0 & 0 & 0 & 0 & 0 & 0 & 0 & 0 \\
\hline C. glutamicum ATCC 13032 & 0 & 0 & 42 & 0 & 0 & 0 & 0 & 0 & 0 & 0 & 47 \\
\hline C. jeikeium K411 & 0 & 0 & 0 & 0 & 0 & 0 & 0 & 0 & 0 & 45 & 0 \\
\hline C. kroppenstedtii DSM 44385 & 0 & 0 & 0 & 0 & 0 & 0 & 0 & 0 & 0 & 41 & 47 \\
\hline C. urealyticum DSM 7109 & 0 & 0 & 38 & 0 & 0 & 0 & 0 & 0 & 0 & 44 & 41 \\
\hline Nocardioides sp. JS614 & 0 & 0 & 40 & 0 & 0 & 0 & 0 & 0 & 0 & 46 & 46 \\
\hline N. farcinica IFM 10152 & 0 & 0 & 42 & 0 & 0 & 0 & 0 & 0 & 0 & 0 & 44 \\
\hline R. erythropolis PR4 & 0 & 0 & 42 & 0 & 0 & 0 & 0 & 0 & 0 & 42 & 48 \\
\hline R. jostii RHA1 & 0 & 0 & 44 & 0 & 0 & 0 & 0 & 0 & 0 & 41 & 49 \\
\hline R. opacus B4 & 0 & 0 & 44 & 0 & 0 & 0 & 0 & 0 & 0 & 41 & 50 \\
\hline
\end{tabular}


in the 14 NTM species studied (Additional file 1), which did not allow us to design specific mycobacterial primers and probes, according to the rules of primer and probe design (Additional file 3).

DNA sequence alignment of the oxidoreductase and of the small secreted protein did not allow design of PCR primers with a minimal length of 18 oligonucleotides (Additional file 3). Only the DNA sequence alignment of the ATP synthase subunits $C$ allowed designing a PCR primer pair and a probe. We designed the following primers and probe: forward primer FatpE $5^{\prime}$-CGGYGCCGGTATCGGYGA-3' $\left(\mathrm{Tm}=62^{\circ} \mathrm{C}\right)$, with the probe PatpE 5'-ACSGTGATGAAGAACGGBGTRAA-3' $\left(\mathrm{Tm}=68^{\circ} \mathrm{C}\right)$ which might be hydrolyzed by the reverse primer RatpE $5^{\prime}$-CGAAGACGAACARSGCCAT-3' $\quad\left(\mathrm{Tm}=59^{\circ} \mathrm{C}, 182 \mathrm{bp}\right)$.

\section{Real-time PCR validation}

Based on standard curve comparisons, our results showed reproducible amplification signals with similar $\mathrm{Ct}$ values for each genome equivalents of tested mycobacterial strains: $M$. avium, M. fortuitum, $M$. intracellulare, $M$. gordonae, and M. chelonae (Table 2). Detection limit was estimated at about 6 genome equivalents for $M$. chelonae by real-time PCR reaction by testing repetition of dilution limits (i.e. EC95 value: more than $95 \%$ of positive detection for these genome concentration) whereas quantification limits were estimated at about 100 genome equivalents. In the positive collection all 31 mycobacteria species were positively detected by the real-time PCR method. This collection includes NTM species, leprae species and MTC species as tuberculosis and bovis (Table 3). None of the non-mycobacterial environmental strains and none of the CNM collection strains [17], were detected before the end of the 40 PCR cycles (Table 3). These results indicate a sensibility of $100 \%(31 / 31)$ and a specificity of $100 \%(0 / 30)$.

\section{Environmental analyses}

In order to compare with culture-based method (Method A) [28], and evaluate the impact of extraction methods on the quantification process by the new real-time PCR, we used two DNA extraction procedures (Method B and C) on water distribution samples: a commercial kit (Method B) and a published phenol-chloroform extraction (Method
C) [29]. DNA extraction from tap water significantly influenced the result of mycobacteria detection by atpE realtime PCR (Figure 3A). Detection levels from DNA extracted by the kit (Method B) were significantly higher (Wilcoxon signed-rank test, $\mathrm{n}=90, \mathrm{p}=0.002$ ) than those from DNA extracted by phenol/chloroform procedure (Method C). The percentage of positive samples was significantly higher (Chi-square test, $\mathrm{n}=180, \mathrm{df}=1, \mathrm{p}=0.021$ ) when performing the real-time PCR with the DNA extracted by method B (33/90), compared to method C (19/ 90). In order to evaluate the new real-time PCR method, we compared the levels of mycobacteria detected in water distribution samples with a published culture method called method A [28]. Using the method A, Mycobacterium spp. colonies were obtained from $76 \%$ of tap water samples.

Mycobacteria quantification in lake samples by real-time PCR targeting atpE gene, shows a vast diversity of mycobacteria concentration, ranging from $10^{4}$ to $10^{6} \mathrm{ge} / \mathrm{L}$ in water column and neuston samples, and $10^{5}$ to $10^{6} \mathrm{ge} / \mathrm{g}$ DW (dry weight) in sediment samples. Comparison with the previously published methods targeting 16S rRNA [17] shows a high correlation between the results (Figure 3B, Correlation test, $\mathrm{n}=30, \mathrm{Rs}=0.571, \mathrm{p}=0.028$ ).

\section{Discussion}

Although $g y r A, \operatorname{gyr} B, h s p 65, \operatorname{rec} A, r p o B$, and $\operatorname{sod} A$ genes are appropriate for identification purposes [3,4], our results emphasized that these genes seem inappropriate for specific detection of mycobacteria. Indeed, their high similarities with non-mycobacterial genes make specific target design delicate. These new results are in accordance with our previous observations that the molecular targets which were designed based on $\operatorname{gyr} B$ [18], rроB [19] or hsp65 [20] genes, had low specificity [17]. For example, the non-related Helicobacter pylori show positive amplification with several Mycobacterium specific primer pairs [17]. Prospection for more specific targets in mycobacterial genomes seems consequently necessary in order to improve current detection tools based on proteins and/or DNA. The new atpE real-time PCR method that we propose is just as specific, but more sensitive than the previously proposed rrs real-time PCR method which cannot detect some mycobacterial species [17].

Table 2 Characteristics of Mycobacterium avium, M. fortuitum, M. intracellulare, and $M$. chelonae DNA amplification using real-time PCR targeting atpE gene (locus Rv1305 in M. tuberculosis genome)

\begin{tabular}{|c|c|c|c|c|c|}
\hline Real-time PCR characteristics & M. avium & M. fortuitum & M. intracellulare & M. gordonae & M. chelonae \\
\hline Correlation coefficient $r^{2}(\%)$ & 93.4 & 97.4 & 98.1 & 99.6 & 99.8 \\
\hline Efficiencies (\%) & 119 & 109 & 119 & 97 & 101 \\
\hline QL (ge/reaction) & $<100$ & $<100$ & $<100$ & ND & $<100$ \\
\hline DL (95\%) (ge/reaction) & ND & ND & ND & ND & 6 \\
\hline
\end{tabular}

Ct (cycle threshold) set at 0.02. ND stands for not determined, QL for Quantification Limit, and DL for Detection Limit. 
Table 3 Detection of the atpE gene (locus Rv1305 in $M$. tuberculosis genome) in different Mycobacterium species ( $25 \pm 15 \mathrm{ng}$ of DNA) and non-mycobacterial microorganisms (50 $\pm 15 \mathrm{ng}$ of DNA)

\begin{tabular}{|c|c|c|c|}
\hline & $\begin{array}{c}\text { Microorganism } \\
\text { codification }^{\mathrm{a}}\end{array}$ & Microorganism & Results \\
\hline \multirow[t]{31}{*}{$\bar{A}$} & CPS MC13 & M. arupense & Detected \\
\hline & CPS MC11 & M. austroafricanum & Detected \\
\hline & ATCC $25291^{\top}$ & M. avium subsp. avium & Detected \\
\hline & CIP 1173/P2 & M. bovis (BCG) & Detected \\
\hline & ATCC $19977^{\top}$ & M. chelonae spp. abscessus & Detected \\
\hline & ATCC $35752^{\top}$ & M. chelonae spp. chelonae & Detected \\
\hline & CIP $105388^{\top}$ & M. gadium & Detected \\
\hline & ATCC $14470^{\top}$ & M. gordonae & Detected \\
\hline & ATCC $6841^{\top}$ & M. fortuitum spp. fortuitum & Detected \\
\hline & CPS MC8 & M. insubricum & Detected \\
\hline & ATCC $15985^{\top}$ & M. intracellulare & Detected \\
\hline & ATCC $12478^{\top}$ & M. kansasii & Detected \\
\hline & CIP $105465^{\top}$ & M. lentiflavum & Detected \\
\hline & THAI 53 & M. leprae & Detected \\
\hline & CPS MC10 & M. llatzerense & Detected \\
\hline & ATCC $927^{\top}$ & M. marinum & Detected \\
\hline & CIP $105223^{\top}$ & M. mucogenicum & Detected \\
\hline & CIP $106811^{\top}$ & M. nonchromogenicum & Detected \\
\hline & CPS MC6 & M. psychrotolerans & Detected \\
\hline & ATCC $14467^{\top}$ & M. peregrinum & Detected \\
\hline & CPS MC9 & M. porcinum & Detected \\
\hline & CIP $105416^{\top}$ & M. scrofulaceum & Detected \\
\hline & CPS MC7 & M. setense & Detected \\
\hline & ATCC $25275^{\top}$ & M. simiae & Detected \\
\hline & ATCC $19420^{\top}$ & M. smegmatis & Detected \\
\hline & ATCC $35799^{\top}$ & M. szulgai & Detected \\
\hline & CIP $104321^{\top}$ & M. terrae & Detected \\
\hline & CIP 106368 & M. tusciae & Detected \\
\hline & ATCC $25618^{\top}$ & M. tuberculosis (H37Rv) & Detected \\
\hline & CPS CR08085632 & M. ulcerans & Detected \\
\hline & ATCC $19250^{\top}$ & M. xenopi & Detected \\
\hline \multirow[t]{10}{*}{ B } & CMR SC10 & Acinetobacter sp. & ND \\
\hline & CMR SC9 & Aeromonas sp. & ND \\
\hline & CMR SC23 & Arthrobacter sp. & ND \\
\hline & CMR SC44 & Aspergillus sp. & ND \\
\hline & CMR SC5 & Bacillus sp. & ND \\
\hline & CMR SC24 & Brevundimonas sp. & ND \\
\hline & ATCC $6871^{\top}$ & C. ammoniagenes & ND \\
\hline & ATCC $13032^{\top}$ & C. glutamicum & ND \\
\hline & ATCC $10700^{\top}$ & C. pseudodiphtheriticum & ND \\
\hline & CMR SC35 & Escherishia sp. & ND \\
\hline
\end{tabular}

Table 3 Detection of the atpE gene (locus Rv1305 in $M$. tuberculosis genome) in different Mycobacterium species ( $25 \pm 15 \mathrm{ng}$ of DNA) and non-mycobacterial microorganisms (50 $\pm 15 \mathrm{ng}$ of DNA) (Continued)

\begin{tabular}{ccc}
\hline CMR SC19 & Flavobacterium sp. & ND \\
ATCC 43504 & Helicobacter pylori & ND \\
CMR SC45 & Kocuria sp. & ND \\
CMR SC31 & Leuclercia sp. & ND \\
CMR SC28 & Leucobacter sp. & ND \\
CMR SC29 & Microbacterium sp. & ND \\
CMR SC3 & Micrococcus sp. & ND \\
DSM 44546 & N. cerradoensis & ND \\
DSM 44490 & N. cummidelens & ND \\
IFM 10152 & N. farcinica & ND \\
CMR SC42 & Penicillium sp. & ND \\
CMR SC1 & Pseudomonas sp. & ND \\
CMR SC26 & Rhodococcus sp. & ND \\
CMR SC34 & Serracia fonticola & ND \\
CMR SC22 & Solibacillus sp. & ND \\
CMR SC12 & Staphylococcus caprae & ND \\
CMR SC6 & Staphylococcus hominis & ND \\
CMR SC46 & Staphylococcus lugdunensis & ND \\
CMR SC49 & Streptomyces sp. & ND \\
CMR SC41 & Trichoderma sp. & ND \\
\hline
\end{tabular}

TaqMan $^{\oplus}$ real-time $\mathrm{PCR}$ amplification was performed using forward primer FatpE, reverse primer RatpE and probe PatpE in duplicate assays. ND stands for not detected sigmoidal curve.

${ }^{a}$ ATCC: American Type Culture Collection; CPS: Collection de la Pitié-

Salpêtrière, Paris, France; 'T: type strain; CIP: Collection de l'Institut Pasteur, Paris, France; CMR: Collection de Microorganismes de Radomski et al. 2010 [17], Paris, France; DSM: DSMZ-Deutsche Sammlung von Mikroorganismen und Zellkulturen, Braunschweig, Germany; IFM: Culture Collection of the Research Centre for Pathogenic Fungi and Microbial Toxicoses, Chiba, Japan.

The proposed strategy is aimed at comparing mycobacterial and non-mycobacterial genomic proteins to reference genomic DNA of $M$. tuberculosis $\mathrm{H} 37 \mathrm{Rv}$, sorting proteins according to similarity requests and listing candidate proteins (Figure 1). We chose to perform protein-level comparisons in order to identify exclusively conserved proteins in Mycobacterium spp. because non-coding regions, as intergenic regions and insertion sequences, are known to be less conserved than coding regions in $M$. tuberculosis genomes [30]. According to literature, our results emphasized that almost half of the $M$. tuberculosis $\mathrm{H} 37 \mathrm{Rv}$ predicted proteins are potentially present in the genomes of $\mathrm{CNM}$ group members. More precisely, mycobacteria belong to Actinobacteria which may explain the presence of 48 to $73 \%$ shared genes among high $\mathrm{G}+\mathrm{C}$ content microorganisms [31-34]. In addition, horizontal gene transfers from different bacteria widely present in soil or water, especially Rhodococcus sp., Nocardia sp. and Streptomyces sp. were previously considered to have happened in the 
Mycobacterium genus which may also explain the shared proteins with non-mycobacterial species [24,27,35]. These observations show that CNM group members must be taken into account in order to develop highly specific mycobacterial targets, considering that these bacteria are commonly found in aquatic and terrestrial environments [36,37].

Our study showed that 11 proteins exclusively conserved in the 16 mycobacterial genomes studied could be selected using our genome comparison strategy (i.e. proteins coded by atpE, atpB, cmaA1, lppM, PE5, $P P E 48, e s x G, e s x H$ and esxR genes, as well as an oxidoreductase and a small secreted protein). Only the aptE gene could be used to design primers and a probe for mycobacteria detection. Concerning the other genes, the sequence polymorphism among NTM species did not allow designing molecular targets for Mycobacterium spp. detection. However, these genes could be of immunological or pathogenic importance. Indeed, PE and PPE family proteins represent 0.9 to $4.2 \%$ of the genome coding capacity of several mycobacteria [22,25,26,35], and are suspected to play a major antigenic role in immune response [38]. PE and PPE family proteins are often associated with mycobacterial es $x$ gene clusters, which encode ATP dependent specific secretion system [24] and are required to export specific members of the $6-\mathrm{kDa}$ early secreted antigenic target (ESAT-6) protein family [26]. Together, ATP dependent specific secretion system and ESAT- 6 protein family play a major role in the virulence and life cycle of mycobacteria [24,26]. Nevertheless, PE and PPE family proteins, and proteins coded by es $x$ gene clusters are very small and polymorphous among genomes of the 11 NTM species compared (Table 1). Mycobacterial cell wall is also important in pathology, and could procure interesting PCR targets. For instance, several studies emphasized that cyclopropanation of the mycolic acids is common among pathogenic mycobacteria but rare among saprophytic species [39]. Although having sufficient length, proteins CMAS coded by the cmaA1 gene and lipoprotein coded by lppM gene in $M$. tuberculosis $\mathrm{H} 37 \mathrm{Rv}$, were also polymorphous among genomes of the 11 NTM species compared (Table 1) and thus could not be used to design a primer pair and a probe (Additional file 2). Nevertheless, polymorphism of mycobacterial mycolic acids is useful for mycobacteria identification [40,41].

The $a t p E$ gene which codes ATP synthase subunit C in $M$. tuberculosis $\mathrm{H} 37 \mathrm{Rv}$ genome (locus Rv1305) is exclusively conserved in the genomes of the 17 mycobacterial species studied (Additional file 2), and its length and relative conservation among mycobacteria make it an adequate molecular target in order to detect $\mathrm{Myco}$ bacterium genus. It is remarkable to see that the protein coded by atpE gene was also the target of the new antimycobacterial compound recently described: diarylquinoline R207910 [42]. This compound shows a specific bactericidal effect on mycobacteria and none in other genera [43]. In addition, our in vitro results demonstrated the specificity of the atpE gene (locus Rv1305), which codes for the ATP synthase protein subunit C. These results also showed that our strategy of target design based on MycoHit software (Figure 1) gave very useful results for designing highly specific primers and might be applied to other microorganism clusters.

In vitro validation of the real-time PCR targeting the $a t p E$ gene showed a very high specificity and sensitivity, as well as reproducible quantification of different mycobacteria species. The new real-time method was tested on a realistic number of mycobacterial species including several slow and rapid growing NTM, although not all the described mycobacterial species were tested. In addition, application of this real-time PCR method to environmental samples showed that Mycobacterium was detected in tap water samples. The discrepancy between the cultural and molecular techniques was previously described for other pathogens, and the lower level of prevalence obtained by the PCR methods was probably due to our concentration and extraction procedures. These protocol steps must be improved to detect low level of NTM even if the used spin column seemed more appropriate for DNA extraction from environmental samples compared to classical phenol-chloroform extraction. Moreover, culture method did not detect higher level of mycobacterial cells compared to the molecular one. Both methods have advantages and drawbacks, and it may explain the differences observed. For instance, molecular methods could detect dead bacteria, or viable but uncultivable bacteria. However, the real-time PCR targeting the atpE gene allows more accurate Mycobacterium spp. quantification, contrary to culture based method which is subjected to many drawbacks such as decontamination artifact (about $2 \log _{10}$ reduction for $M$. chelonae), slow mycobacteria growth, clumping of mycobacterial cells, high hydrophobicity of mycobacteria and contamination of culture media by other fast growing environmental microorganisms [44].

Comparison of the method targeting atpE with previously described method targeting $16 \mathrm{~S}$ rRNA, [17], showed a high correlation. Moreover the method targeting atpE gene presents two major advantages over the method targeting rrs gene. First, the new method detects all the tested mycobacterial strains, while the method targeting $r r s$ gene cannot detect isolates of $M$. celatum, $M$. heckeshornense, and M. leprae [17]. Second, the atpE gene is present in a single copy in the Mycobacterium genomes, while the $16 \mathrm{~S}$ rRNA gene is present either in 1 or 2 copies in the genome [45]. When comparing samples it will be simpler to interpret the data with a stable 
gene copy number, and probably give a better accuracy of the mycobacterial concentration.

One of the limitations of this study is that only 31 mycobacterial species were tested in vitro as positive controls whereas more than 150 mycobacterial species have been described so far [1]. To date, we have confirmed the sensitivity of the atpE real-time PCR method using a large representative collection of mycobacterial species (31 species, e.g. around 20\% of described species), including members of MTC $(\mathrm{n}=2)$, M. leprae species $(\mathrm{n}=1)$, slow growing NTM $(\mathrm{n}=13)$, and rapid growing NTM $(n=15)$. Given the broad diversity of mycobacterial species we have tested in this study, we expect the method to be applicable to all species within the Mycobacterium genus. In addition, it is the first time that a sensitive and specific molecular target has been identified based on an in silico comparison of 16 mycobacterial (13 species) and 12 non-mycobacterial genomes (4 closely related species).

\section{Conclusions}

In conclusion, although our strategy did not take into account non-coding regions, such as insertion sequences, repetitive units, non-functional RNA, and structural ribosomal RNAs, the comparison of whole bacterial genomes for design of specific primers is a promising approach not only for mycobacteria but also for other cultured bacterial or archaeal groups for which whole sequenced genomes are accumulating in databases. Metagenomic libraries from environmental samples which are increasingly performed in microbial ecology studies [46] could also provide useful data for the design of specific targets toward uncultured Bacteria and Archaea. Using these databases, MycoHit, or other new software, may then be used to design new primers for real-time PCR detection or quantification, for in situ hybridization and other molecular tools. With this approach we were able to design primer pairs and a probe that target specific mycobacterial atpE gene, and could be used to detect and quantify very specifically mycobacteria in environmental samples. Although the $a t p E$ gene may not be appropriate for microdiversity studies, it appeared to be very useful for specific detection of the genus Mycobacterium in environmental samples. More generally, genome comparison used here showed its utility to identify specific genera's targets, and could be used to identify specific proteins for antimicrobial design as previously emphasized [47].

\section{Methods}

\section{In silico comparison strategy}

In order to detect $M$. tuberculosis genes, presenting homologue genes in other mycobacterial genomes, and not presenting homologue genes in non-mycobacteria genomes, we used the MycoHit software version 14.17 (Zipped copy of the files and instructions for this application are available in the Behr Research Lab, https://www.mcgill.ca/molepi/) and performed an alignment search with Stand Alone tblastn algorithm as previously described [27]. Stand Alone tblastn algorithm has been chosen because coding sequences are known to be more conserved in mycobacterial genomes than non-coding sequences, as intergenic regions, insertion sequences, or phage sequences [30]. Genome of $M$. tuberculosis $\mathrm{H} 37 \mathrm{Rv}$ has been used as a reference of the Mycobacterium genus, because it is the most historically described mycobacterial genome [22]. Based on the 3989 predicted proteins from M. tuberculosis H37Rv, corresponding to the query sequences used in order to search for matches in the genomic DNA of other organisms (Figure 1), a matrix of 107703 scores (3989 protein sequences blasted against 12 non-mycobacterial
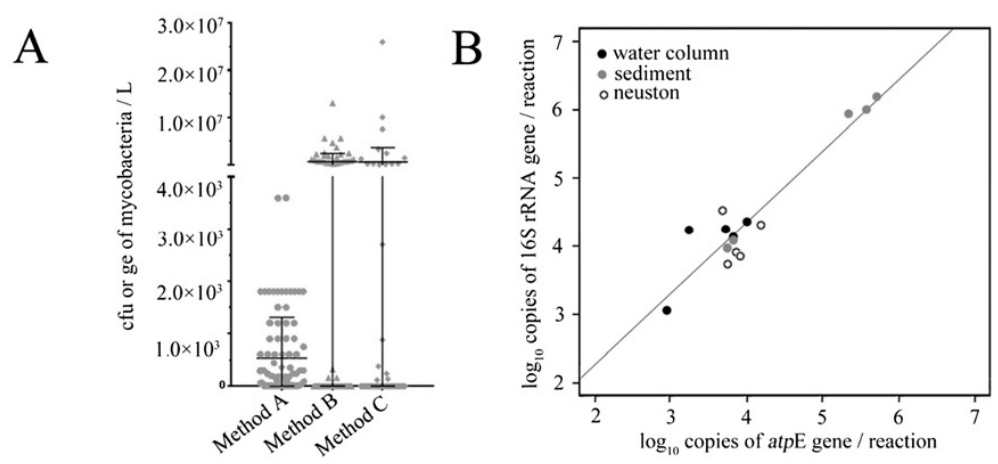

Figure 3 Mycobacteria quantification in environmental samples and comparison to reference methods. A) Quantification in drinking water samples ( $n=90)$ was performed by culture method (Method A: Le Dantec et al. 2002) [28], and the new real-time PCR targeting the atpE gene (locus Rv1305 in M. tuberculosis genome) applied to DNA extracted by commercial spin column procedure (Method B: Qiagen kit extraction), or reference DNA extraction procedure (Method C: Radomski et al. 2011) [29]. B) Quantification in lake samples ( $\mathrm{n}=15$ ) was performed measured by real-time PCR targeting 165 rRNA (Radomski et al. 2010) [17] or atpE genes. 
genomes and 15 mycobacterial genomes) was obtained. As previously described [27] and according to NCBI procedures [48], expected value was set at $\mathrm{e}^{-10}$. Following sequence comparisons, the MycoHit software allowed to sort scores according to similarity requests which were performed on the one hand toward mycobacterial genomes, and on the other hand toward non-mycobacterial genomes (Figure 1). A protein list of the reference target, which can be downloaded from NCBI web site (http:// www.ncbi.nlm.nih.gov), allowed identification of the conserved mycobacterial proteins presenting no homology in non-mycobacterial genomes (Figure 1).

\section{Mycobacterial genome database}

In order to perform comparisons of pathogenic $(\mathrm{P})$ and non-pathogenic (NP) mycobacterial genomes with $M$. tuberculosis $\mathrm{H} 37 \mathrm{Rv}$ genome using MycoHit software, sequences were obtained at NCBI web site (http:// www.ncbi.nlm.nih.gov) using the accession numbers: M. abscessus ATCC 19977 (CU458896.1) (P), M. avium 104 (CP000479.1) (P), M. avium subsp. paratuberculosis K10 (AE016958.1) (P), M. bovis subsp. bovis AF2122/97 (BX248333.1) (P), M. gilvum PYR-GCK (CP000656.1) (NP), M. marinum M (CP000854.1) (P), M. smegmatis MC2 155 (CP000480.1) (NP), Mycobacterium sp. JLS (CP000580.1) (NP), Mycobacterium sp. KMS (CP000518.1) (NP), Mycobacterium sp. MCS (CP000384.1) (NP), $M$. tuberculosis CDC1551 (AE000516.2) (P), M. tuberculosis H37Ra (CP000611.1) (NP), M. tuberculosis H37Rv (AL123456.2) (P), M. tuberculosis KZN 1435 (CP001658.1) (P), M. ulcerans Agy99 (CP000325.1) (P), and M. vanbaalenii PYR-1 (CP000511.1) (P). In order to avoid data lost during genome comparisons performed by MycoHit software, we have chosen to ignore some mycobacterial genomes. Since the number of coding proteins is much lower compared to other mycobacterial species, $M$. leprae Br4923 (FM211192.1) (P), and M. leprae TN (AL450380.1) (P) were ignored in the analysis (e.g. 1604 coding proteins in M. leprae Br4923 or 1605 coding proteins in M. leprae TN, against 6716 coding proteins in M. smegmatis MC2 155) [22,24-26,35]. Genomes of $M$. bovis BCG Pasteur 1173P2 (AM408590.1) (NP) and M. bovis BCG Tokyo 172 (AP010918.1) (NP) were also not taken into account, because these vicinal genomes present mutations [49]. Moreover, genomes of $M$. intracellulare ATCC 13950 (ABIN00000000) (P), M. kansasii ATCC 12478 (ACBV0 0000000) (P) and M. parascrofulaceum BAA-614 (ADNV 00000000) (P) were also not used during MycoHit proceedings, because their genomes were still not assembled at the moment we performed the first screening step of our analysis. Nevertheless, the genomes of M. leprae, M. bovis BCG, M. intracellulare, M. kansasii and M. parascrofulaceum were used during alignment of nucleic sequences of the most conserved proteins in mycobacterial genomes.

\section{Non-mycobacterial genome database}

We selected non-mycobacterial genomes of species from the CNM group using the following accession numbers: Corynebacterium aurimucosum ATCC 700975 (CP0016 01.1), C. diphtheriae NCTC 13129 (BX248353.1), C. efficiens YS-314 (BA000035.2), C. glutamicum ATCC 13032 (BX927147.1), C. jeikeium K411 (NC_007164), C. kroppenstedtii DSM 44385 (CP001620.1), C. urealyticum DSM 7109 (AM942444.1), Nocardia farcinica IFM 10152 (AP006618.1), Nocardioides sp. JS614 (CP000509.1), Rhodococcus erythropolis PR4 (AP008957.1), R. jostii RHA1 (CP000431.1), and R. opacus B4 (AP011115.1).

\section{Primer pair and probe design}

In order to check the homology of the selected mycobacterial sequences, the protein and DNA sequences of these selected proteins were aligned using the ClustalW multiple alignment of the BioEdit software 7.0.9.0 with 1000 bootstraps [50]. Primer pair and probe was designed from the best fitted gene sequences (after protein screening and selection) by visual analysis and using the Beacon Designer software version 7.90 (Premier Biosoft International, Palo Alto, Calif.).

\section{Real-time PCR validation}

Reproducibility, sensitivity and specificity of the new real-time PCR method were estimated using DNA from a previously described microorganism collection, and according to Radomski et al. protocol [17]. Reproducibility, efficiency, limits of detection and quantification of the real-time PCR methods [44] were estimated by quantification of several tenfold dilutions (10 replications of 400 , $100,40,20,4,0.4$ and 0.04 genome equivalent (ge) by reaction) of a known quantity of DNA extracted from four strains: $M$. avium, $M$. fortuitum, $M$. intracellulare and $M$. gordonae (identified from the national French reference laboratory collection). Specificity and sensitivity were estimated against 30 non-mycobacteria (negative) strains and 31 mycobacteria (positive), respectively. The collection contained reference and environmental strains of mycobacteria, as well as, strains of the closely related CNM group, and other non-actinobacteria strains isolated from the environment [17]. Mycobacteria collection included MTC $(n=2)$ and leprae species $(n=1)$, as well as species of slow growing NTM $(\mathrm{n}=13)$, and rapid growing NTM $(\mathrm{n}=15)$. TaqMan ${ }^{\oplus}$ real-time PCR were performed in duplicate using an ABI7500 real-time PCR system (Applied Biosystems), a Lifetech 7500 software version 2.0.6 (Applied Biosystems) and TaqMan fast virus 1-STEP Master Mix with 6-carboxy-X-rhodamine (ROX) (Applied Biosystems). The TaqMan ${ }^{\circ}$ probes were labeled (Eurogentec) with the fluorescent dyes 6-carboxyfluorescein ( $5^{\prime}$ end) and Black Hole Quencher ( $3^{\prime}$ end). All reactions were performed in a $25 \mu \mathrm{l}$ reaction mixture volume (2.5 $\mu \mathrm{l}$ of DNA) 
with $500 \mathrm{nM}$ of forward primer, $500 \mathrm{nM}$ of reverse primer, $50 \mathrm{nM}$ of probe and $5 \mathrm{mM}$ of $\mathrm{MgCl}_{2}$. Reverse transcriptase was inactivated immediately $\left(95{ }^{\circ} \mathrm{C}, 45 \mathrm{~s}\right)$ according to the manufacturer instruction, and real-time PCR consisted in 40 cycles of denaturation $\left(95^{\circ} \mathrm{C}\right.$ for $\left.3 \mathrm{~s}\right)$, annealing and extension (both steps at $60^{\circ} \mathrm{C}$ for $30 \mathrm{~s}$ ). Determinations of cycle threshold were performed by setting the instrument's threshold line at $0.02 \Delta \mathrm{Rn}$ units (fluorescence gain above the baseline divided by the ROX channel signal).

\section{Environmental analyses}

In order to compare the new real-time PCR method to the culture method, 26 tap water distribution points in Paris (France) were sampled between April 2011 and July 2011, corresponding to 90 samples. Briefly, one liter of tap water was sampled in sterile plastic bottle, then centrifuged at $5000 \times \mathrm{g}$ for $2 \mathrm{~h}$ and finally re-suspended in $1 \mathrm{ml}$ of water. Mycobacteria density was estimated by culture (Method A) in all these samples following the procedure previously described by Le Dantec et al. [28]. In parallel, DNA was extracted using two different methods: i) a bacterial DNA extraction kit (QIAamp DNA mini kit, Qiagen) according to the manufacturer recommendations (Method B), and ii) a phenolchloroform extraction procedure according to Radomski et al. [29] (Method C). Extracted DNA was 10 fold diluted and mycobacteria density was estimated in duplicate using the new real-time PCR method.

Using environmental samples, the new atpE targeting method was also compared a previously described $r$ rs targeting method [17]. More precisely, samples collected from water column, sediment, and neuston of two urbanized lakes (Daumesnil Lake, Paris, France, and Créteil Lake, Créteil, France) were analyzed in triplicate. Water samples (column and neuston) were centrifuged $1 \mathrm{~h}$ at $7500 \times \mathrm{g}$, and DNA was extracted using a MagNA Pure System (Roche). Sediment samples were lyophilized and DNA was isolated using FastDNA SPIN kit for Soil according to the manufacturer's instructions (MP Biomedicals, Santa Ana, CA). Statistical analyses were carried out using R software v. 2.15 [51].

\section{Availability of supporting data}

The data sets supporting the results of this article are included within the article and its additional files.

\section{Additional files}

Additional file 1: Similarities (\%) between Mycobacterium tuberculosis H37Rv (AL123456.2) proteins and proteins of targeted mycobacterial genomes and proteins of non-targeted genomes. Targeted mycobacterial genomes include M. tuberculosis H37Ra (CP000611.1), M. tuberculosis CDC 1551 (AE000516.2), M. tuberculosis KZN 1435 (CP001658.1), M. bovis AF2122/97 (BX248333.1), M. ulcerans Agy99 (CP000325.1), M. marinum M (CP000854.1), M. avium 104 (CP000479.1), M. paratuberculosis K10 (AE016958.1), M. smegmatis MC2 155 (CP000480.1), M. abscessus ATCC 19977 (CU458896.1), M. gilvum PYG-GCK (CP000656.1), M. vanbaalenii PYR-1 (CP000511.1), Mycobacterium sp. JLS (CP000580.1), Mycobacterium sp. KMS (CP000518.1), Mycobacterium sp. MCS (CP000384.1), and non-targeted genomes include Corynebacterium aurimucosum ATCC 700975 (CP001601.1), C. diphteriae NCTC 13129 (BX248353.1), C. efficiens YS-314 (BA000035.2), C. glutamicum ATCC 13032 (BX927147.1), C. jeikeium K411 (NC_007164), C. kroppenstedtii DSM 44385 (CP001620.1), C. urealyticum DSM 7109 (AM942444.1), Nocardia farcinica IFM 10152 (AP006618.1), Nocardioides sp. JS614 (CP000509.1), Rhodococcus erythropolis PR4 (AP008957.1), R. jostii RHA1 (CP000431.1) and R. opacus B4 (AP011115.1).

Additional file 2: Protein sequence alignment of conserved proteins in mycobacterial genomes. Sequences are from genomes of M. abscessus ATCC 19977 (CU458896.1), M. avium 104 (CP000479.1), M. avium subsp. paratuberculosis K10 (AE016958.1), M. bovis subsp. bovis AF2122/97 (BX248333.1), M. bovis BCG Pasteur 1173P2 (AM408590.1), M. bovis BCG Tokyo 172 (AP010918.1), M. gilvum PYR-GCK (CP000656.1), M. intracellulare ATCC 13950 (ABIN00000000), M. kansasii ATCC 12478 (ACBV00000000), M. leprae Br4923 (FM211192.1), M. leprae TN (AL450380.1), M. marinum M (CP000854.1), M. parascrofulaceum BAA-614 (ADNV00000000), M. smegmatis MC2 155 (CP000480.1), Mycobacterium sp. JLS (CP000580.1), Mycobacterium sp. KMS (CP000518.1), Mycobacterium sp. MCS (CP000384.1), M. tuberculosis CDC1551 (AE000516.2), M. tuberculosis H37Ra (CP000611.1), M. tuberculosis H37Rv (AL123456.2), M. tuberculosis KZN 1435 (CP001658.1), M. ulcerans Agy99 (CP000325.1) and M. vanbaalenii PYR-1 (CP000511.1).

Additional file 3: DNA sequence alignment of conserved proteins in mycobacterial genomes. Sequences are from genomes of $M$. abscessus ATCC 19977 (CU458896.1), M. avium 104 (CP000479.1), M. avium subsp. paratuberculosis K10 (AE016958.1), M. bovis subsp. bovis AF2122/97 (BX248333.1), M. bovis BCG Pasteur 1173P2 (AM408590.1), M. bovis BCG Tokyo 172 (AP010918.1), M. gilvum PYR-GCK (CP000656.1), M. intracellulare ATCC 13950 (ABIN00000000), M. kansasii ATCC 12478 (ACBV00000000), M. leprae Br4923 (FM211192.1), M. leprae TN (AL450380.1), M. marinum M (CP000854.1), M. parascrofulaceum BAA-614 (ADNV00000000), M. smegmatis MC2 155 (CP000480.1), Mycobacterium sp. JLS (CP000580.1), Mycobacterium sp. KMS (CP000518.1), Mycobacterium sp. MCS (CP000384.1), M. tuberculosis CDC1551 (AE000516.2), M. tuberculosis H37Ra (CP000611.1), M. tuberculosis H37Rv (AL123456.2), M. tuberculosis KZN 1435 (CP001658.1), M. ulcerans Agy99 (CP000325.1) and M. vanbaalenii PYR-1 (CP000511.1).

\section{Abbreviations}

ATP: Adenosine triphosphate; CMAS: Cyclopropane mycolic acid synthase; CNM: Corynebacterium, Nocardia, Rhodococcus, Mycobacterium; ITS: Internal transcribed spacer; MTC: Mycobacterium tuberculosis complex; NP: Nonpathogenic; NTM: Non-tuberculous mycobacteria; P: Pathogenic.

\section{Competing interests}

The authors declare that they have no competing interests.

\section{Authors' contributions}

NR contributed in the experimental design, data acquisition and interpretation under the supervisions of $F \mathrm{~L}, \mathrm{RM}, \mathrm{EC}$, and $\mathrm{LM}$, and was involved in writing the manuscript. For in silico comparisons, FJV provided technical assistance and MAB supervised data interpretation. HA and LM carried out the in vitro assays, and participated to statistical analyses and manuscript writing. All authors read and approved the manuscript.

\section{Acknowledgements}

We thank Pr. Jacques Printems from the laboratory of analysis and applied mathematics (CNRS UMR 8050) in Paris Est University for access to his computer (MacPro3.1, Quad-Core Intel Xeon) in order to perform tblastn algorithms, which run between 1 and 80 hours for each genome comparison according to the similarity levels with the reference genome. We also thank members of the R\&D Biology lab from Eau de Paris, Claire Therial from LEESU, as well as, Michael Reed and Lynn Dery Capes from the Research Institute of the McGill University Health Centre. 


\section{Author details}

'Laboratoire Eau Environnement Systèmes Urbains (Leesu) UMR MA 102-AgroParisTech, Université Paris-Est, 6-8 avenue Blaise Pascal Cité Descartes, FR 77455, Champs sur Marne, France. ${ }^{2}$ Research Institute of the McGill University Health Centre, 1650 Cedar Avenue, Montreal H3G 1A4, QC, Canada. ${ }^{3}$ Département Infection et Epidémiologie, Infections Bactériennes Invasives, Institut Pasteur, 28 Rue du Dr. Roux, Paris, F 75015, France. ${ }^{4}$ Laboratoire associé du Centre national de référence des mycobactéries et de la résistance aux antituberculeux, AP-HP, Groupe Hospitalier Saint Louis-Lariboisière, Université Paris Diderot EA3964, FR 75475, Paris, France. ${ }^{5}$ Eau de Paris, Direction Recherche et Développement Qualité de l'Eau (DRDQE), 33 avenue Jean Jaurès, FR 94200, Ivry-sur-Seine, France.

Received: 15 March 2013 Accepted: 26 November 2013

Published: 3 December 2013

\section{References}

1. Kazda J: The chronology of mycobacteria and the development of mycobacterial ecology. In The ecology of mycobacteria: Impact on animal's and human's health, Volume 1. Edited by Kazda J, Pavlik I, Falkinham JO, Hruska K. Dordrecht Heidelberg London New York: Springer; 2009:1-11.

2. Radomski N, Cambau E, Moulin L, Haenn S, Moilleron R, Lucas FS: Comparison of culture methods for isolation of nontuberculous mycobacteria from surface waters. Appl Environ Microbiol 2010, 76(11):3514-3520

3. Adékambi T, Drancourt M: Dissection of phylogenetic relationships among 19 rapidly growing Mycobacterium species by $16 \mathrm{~S}$ rRNA, hsp65, sodA, recA and rpoB gene sequencing. Int I Syst Evol Microbiol 2004, 54(6):2095-2105

4. Gomila M, Ramirez A, Lalucat J: Diversity of environmental Mycobacterium isolates from hemodialysis water as shown by a multigene sequencing approach. Appl Environ Microbiol 2007, 73(12):3787-3797.

5. Mendum TA, Chilima BZ, Hirsch PR: The PCR amplification of nontuberculous mycobacterial $16 \mathrm{~S}$ rRNA sequences from soil. FEMS Microbiol Lett 2000, 185(2):189-192.

6. Garcia-Quintanilla A, Gonzalez-Martin J, Tudo G, Espasa M, Jiménez de Anta MT: Simultaneous identification of Mycobacterium genus and Mycobacterium tuberculosis complex in clinical samples by $5^{\prime}$-exonuclease fluorogenic PCR. J Clin Microbiol 2002, 40(12):4646-4651.

7. Nieminen T, Pakarinen J, Tsitko I, Salkinoja-Salonen M, Breitenstein A, AliVehmas T, Neubauer P: 16S rRNA targeted sandwich hybridization method for direct quantification of mycobacteria in soils. J Microbiol Methods 2006, 67(1):44-55.

8. Dutil S, Veillette M, Mériaux A, Lazure L, Barbeau J, Duchaine C: Aerosolization of mycobacteria and legionellae during dental treatment: Low exposure despite dental unit contamination. Environ Microbiol 2007, 9(11):2836-2843.

9. Böddinghaus B, Rogall T, Flohr T, Blocker H, Bottger EC: Detection and identification of mycobacteria by amplification of rRNA. J Clin Microbiol 1990, 28(8):1751-1759

10. Zolg JW, Philippi-Schulz S: The superoxide dismutase gene, a target for detection and identification of mycobacteria by PCR. J Clin Microbiol 1994, 32(11):2801-2812.

11. Pryor M, Springthorpe S, Riffard S, Brooks T, Huo Y, Davis G, Sattar SA: Investigation of opportunistic pathogens in municipal drinking water under different supply and treatment regimes. Water Sci Technol 2004 50(1):83-90.

12. Niva M, Hernesmaa A, Haahtela K, Salkinoja-Salonen M, Sivonen K, Haukka K: Actinobacteria communities of borel forest soil and lake water are rich in mycobacteria. Boreal Environ Res 2006, 11(1):45-53.

13. Leys NM, Ryngaert A, Bastiaens L, Wattiau P, Top EM, Verstraete W, Springael D: Occurrence and community composition of fast-growing Mycobacterium in soils contaminated with polycyclic aromatic hydrocarbons. FEMS Microbiol Ecol 2005, 51(3):375-388.

14. Uyttebroek M, Vermeir S, Wattiau P, Ryngaert A, Springael D: Characterization of cultures enriched from acidic Polycyclic Aromatic Hydrocarbon-contaminated soil for growth on pyrene at low pH. Appl Environ Microbiol 2007, 73(10):3159-3164.

15. Uyttebroek M, Breugelmans $P$, Janssen $M$, Wattiau $P$, Joffe $B$, Karlson $U$, Ortega-Calvo JJ, Bastiaens L, Ryngaert A, Hausner M, et al: Mycobacterium community and polycyclic aromatic hydrocarbons (PAHs) among different size fractions of a long-term PAH-contaminated soil. Environ Microbiol 2006, 8(5):836-847.

16. Uyttebroek M, Spoden A, Ortega-Calvo JJ, Wouters K, Wattiau P, Bastiaens L, Springael D: Differential responses of Eubacterial, Mycobacterium, and Sphingomonas communities in Polycyclic Aromatic Hydrocarbon (PAH)contaminated soil to artificially induced changes in PAH profile. J Environ Qual 2007, 36(1):1403-1411.

17. Radomski N, Lucas FS, Moilleron R, Cambau E, Haenn S, Moulin L: Development of a real-time qPCR method for detection and enumeration of Mycobacterium spp. in surface water. Appl Environ Microbiol 2010, 76(11):7348-7351.

18. Fukushima M, Kakinuma K, Hayashi H, Nagai H, Ito K, Kawaguchi R. Detection and identification of Mycobacterium species isolates by DNA microarray. J Clin Microbiol 2003, 41(6):2605-2615.

19. Kim BJ, Hong SK, Lee KH, Yun YJ, Kim EC, Park YG, Bai GH, Kook YH: Differential identification of Mycobacterium tuberculosis complex and nontuberculous mycobacteria by duplex PCR assay using the RNA polymerase gene (rpoB). J Clin Microbiol 2004, 42(3):1308-1312.

20. Tobler NE, Pfunder M, Herzog K, Frey JE, Altwegg M: Rapid detection and species identification of Mycobacterium spp. using real-time PCR and DNA-Microarray. J Microbiol Methods 2006, 66(1):116-124.

21. Murray RGE, Brenner DJ, Bryant MP, Holt JG, Krieg NR, Mouldier JW, Pfennig N, Snearth PHA, Staley JT, Lapage SP, et al: Bergey's manual of systematic and bacteriology, Volume 2. 1st edition. Baltimore, USA: Williams and Wilkins; 1989.

22. Cole ST, Brosch R, Parkhill J, Garnier T, Churcher C, Harris D, Gordon SV, Eiglmeier K, Gas S, Barry CE, et al: Deciphering the biology of Mycobacterium tuberculosis for the complete genome sequence. Nat Aust 1998, 44(6685):393-537.

23. Nyrén P: The history of pyrosequencing. Methods Mol Biol 2007, 373(1):1-14.

24. Ripoll F, Pasek S, Schenowitz C, Dossat C, Barbe V, Rottman M, Macheras E, Heym B, Herrmann JL, Daffé M, et al: Non mycobacterial virulence genes in the genome of the emerging pathogen Mycobacterium abscessus. PLoS One 2009, 4(6):1-12.

25. Li L, Bannantine J, Zhang Q, Amonsin A, May B, Alt D, Banerij N, Kanjilal S, Kapur $\mathrm{V}$ : The complete genome sequence of Mycobacterium avium subspecies paratuberculosis. Proc Natl Acad Sci U S A 2005, 102(35):12344-13349.

26. Stinear TP, Seemann T, Harrison PF, Jenkin GA, Davies JK, Johnson PDR, Abdellah Z, Arrowsmith C, Chillingworth T, Churcher C, et al: Insights from the complete genome sequence of Mycobacterium marinum on the evolution of Mycobacterium tuberculosis. Genome Res 2010, 18(1):729-741

27. Veyrier F, Pletzer D, Turenne C, Behr MA: Phylogenetic detection of horizontal gene transfer during the step-wise genesis of Mycobacterium tuberculosis. BMC Evo Biol 2009, 196(8):1-14.

28. Le Dantec C, Duguet JP, Montiel A, Dumoutier N, Dubrou S, Vincent V: Occurrence of mycobacteria in water treatment lines and in water distribution systems. Appl Environ Microbiol 2002, 68(11):5318-5325.

29. Radomski N, Betelli L, Moilleron R, Haenn S, Moulin L, Cambau E, Rocher V, Gonçalves A, Lucas FS: Mycobacterium behavior in wastewater treatment plant, a bacterial model distinct from Escherichia coli and enterococci. Environ Sci Technol 2011, 45(12):5380-5386.

30. Cubillos-Ruiz A, Morales J, Zambrano MM: Analysis of the genetic variation in Mycobacterium tuberculosis strains by multiple genome alignments. BMC Res Notes 2008, 7(1):110-120.

31. Casas Botero AE, Torem ML, Souza de Mesquita LM: Fundamental studies of Rhodococcus opacus as a biocollector of calcite and magnesite. Mine Eng 2007, 20(10):1026-1032.

32. Cocito $C$, Gilot P, Coene $M$, de Kesel M, Poupart P, Vannuffel P: Paratuberculosis. Clin Microbiol Rev 1994, 3(7):328-345.

33. Gangadharam PRJ, Jenkins PA: Mycobacteria, basic acpects vol. 1. New York: International Thomson Publishing; 1998.

34. Prescott LM, Harley JP, Klein DA, Bacq-Calberg CM, Dusart J: Les bactéries : Les Gram-positifs riches en G-C. In Microbiologie, Volume 1. Edited by Prescott J, Harley J, Klein D. Bruxelles: De Boeck Université; 2003:541.

35. Garnier T, Eiglmeier K, Camus JC, Medina N, Mansoor H, Pryor M, Duthoy S, Grondin S, Lacroix C, Monsempe C, et al: The complete genome sequence of Mycobacterium bovis. Proc Natl Acad Sci U S A 2003, 100(13):7877-7882.

36. Goodfellow M, Williams ST: Ecology of actionomycetes. Annu Rev Microbiol 1983, 37:189-216.

37. Rowbotham TJ, Cross T: Ecology of Rhodococcus coprophilus and associated Actinomycetes in fresh water and agriculturl habitats. Microbiol 1977, 100(2):231-240. 
38. Voskuil MI, Schnappinger D, Rutherford R, Liu Y, Schoolnik GK: Regulation of the Mycobacterium tuberculosis PE/PPE genes. Tuberculosis (Edinb) 2004, 84(3-4):256-262.

39. Grogan DW, Cronan JE: Cyclopropane ring formation in membrane lipids of bacteria. Microbiol Mol Biol Rev 1997, 61(4):429-441.

40. Butler WR, Ahearn DG, Kilburn JO: High-Performance Liquid Chromatography of mycolic acids as a tool in the identification of Corynebacterium, Nocardia, Rhodococcus, and Mycobacterium species. J Clin Microbiol 1986, 21(1):182-185.

41. Thibert $L$, Lapierre $S$ : Routine application of high-performance liquid chromatography for identification of mycobacteria. J Clin Microbiol 1993, 31(7):1759-1763.

42. Petrella S, Cambau E, Chauffour A, Andries K, Jarlier V, Sougakoff W: Genetic basis for natural and acquired resistance to the diarylquinoline R207910 in mycobacteria. Antimicrob Agents Chemother 2006, 50(8):2853-2856.

43. Andries K, Verhasselt P, Guillemont J, Göhlmann HWH, Neefs JM, Winkler $H$, van Gestel JV, Timmerman P, Zhu M, Lee E, et al: A diarylquinolone drug active on the ATP synthase of Mycobacterium tuberculosis. Science 2005, 307(5707):223-227.

44. Radomski N, Moilleron R, Lucas FS, Falkinham JO III: Challenges in environmental monitoring of pathogens: Case study in Mycobacterium avium. In Current research, technology and education topics in applied microbiology and microbial biotechnology, Volume 2. Edited by Méndez-Vilas A. Badajoz: Formatex Research Center; 2010:1551-1561.

45. Fogel GB, Collins CR, Li J, Brunk CF: Prokaryotic genome size and SSU rDNA copy number: estimation of microbial relative abundance from a mixed population. Microb Ecol 1999, 38(2):93-113.

46. Riesenfeld CS, Schloss PD, Handelsman J: Metagenomics: genome analysis of microbial communities. Annu Rev Genet 2004, 38(1):525-552.

47. Rosamond J, Allsop A: Harnessing the power of the genome in the search for new antibiotics. Science 2000, 287(5460):1973-1976.

48. Yao J, Lin H, Doddapaneni H, Civerolo EL: nWayComp: a genome-wide sequence comparison tool for multiple strains/species of phylogenetically related microorganisms. In Silico Biol 2007, 7(2):195-200.

49. Mahairas GG, Sabo PJ, Hickey MJ, Singh DC, Stover CK: Molecular analysis of genetic differences between Mycobacterium bovis BCG and virulent $M$. bovis. J Bacteriol 1996, 178(5):1274-1282

50. Hall TA: BioEdit: a user-friendly biological sequence alignment editor and analysis program fo windows 95/98/NT. Nucleic Acids Symp Ser 1999, 41(1):95-98.

51. R Development Core Team: $R$ : a language and environment for statistical computing, R foundation for statistical computing. Vienna, Austria; 2012. http://www.R-project.org

\section{doi:10.1186/1471-2180-13-277}

Cite this article as: Radomski et al: atpE gene as a new useful specific molecular target to quantify Mycobacterium in environmental samples. BMC Microbiology 2013 13:277.

\section{Submit your next manuscript to BioMed Central and take full advantage of:}

- Convenient online submission

- Thorough peer review

- No space constraints or color figure charges

- Immediate publication on acceptance

- Inclusion in PubMed, CAS, Scopus and Google Scholar

- Research which is freely available for redistribution 